\title{
STRATUS, a Phase II Study of Abituzumab in Patients with Systemic Sclerosis-associated Interstitial Lung
}

\section{Disease}

Dinesh Khanna, Donald P Tashkin, Athol U Wells, James R Seibold, Stephen Wax, Cristina Vazquez-Mateo, Patricia Fleuranceau-Morel, Doris Damian, Christopher P Denton

\section{ORCID IDs:}

DK, orcid.org/0000-0003-1412-4453

CPD, orcid.org/0000-0003-3975-8938

DPT: orcid.org/0000-0002-5607-4872

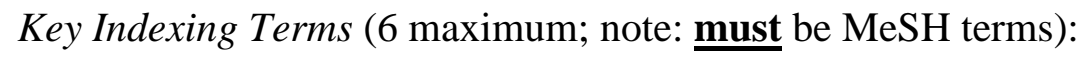

SCLEROSIS; LUNG DISEASES, INTERSTITIAL; INTEGRINS; CLINICAL TRIAL

\section{Affiliations}

From the University of Michigan, Ann Arbor, MI, USA; David Geffen School of Medicine at UCLA, Los Angeles, CA, USA; Royal Brompton Hospital, London, UK; Scleroderma Research Consultants, LLC, Aiken, SC, USA, EMD Serono Inc., * Billerica, MA, USA; University College London Medical School, Royal Free Hospital, London, UK

\section{Funding}

*A business of Merck KGaA, Darmstadt, Germany 
The STRATUS study was sponsored by Merck KGaA (for all study sites residing in countries outside of the USA) or EMD Serono Research and Development Institute, Inc., Billerica, MA,

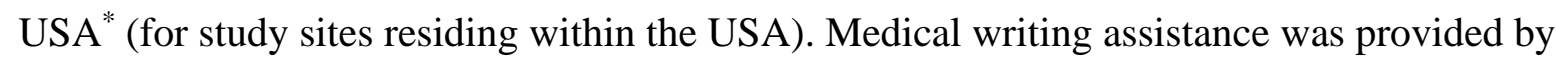
Dan Booth (Bioscript Science, Macclesfield, UK) and funded by Merck KGaA.

\section{Role of the funding source}

The STRATUS study was funded by Merck KGaA, Darmstadt, Germany and EMD Serono Research Institute Inc. (a business of Merck KGaA, Darmstadt, Germany). Merck KGaA authors were involved in the study design, collection, analysis and interpretation of the data, and in the writing of and decision to submit the manuscript.

\section{Conflict of interest}

DK has received consulting fees from Actelion, Acceleron, Arena, Bayer, Boehringer Ingelheim, Bristol-Myer Squibb, CSL Behring, Corbus, Cytori, GSK, Genentech/Roche, Galapagos, Merck, Mitsubishi Tanabi, and UCB. He has received grants (to the University of Michigan) as part of investigator-initiated trials from Bayer, Bristol-Myer Squibb, and Pfizer. DK has stock options in Eicos Sciences, Inc and is an employee of Civi BioPharma, Inc. DPT has received consulting fees from EMD Serono, Boehringer-Ingelheim and Genentech (funder of the Scleroderma Lung Study III).

AUW reports personal fees from Intermune (advisory board and speaker fees), Boehringer Ingelheim (advisory board and speaker fees), Gilead (advisory board fees), MSD (advisory board fees), Roche (advisory board and speaker fees), Bayer (advisory board and speaker fees), and Chiesi (speaker fees).

JRS has received consulting fees from Atlantic, Bayer, Boehringer Ingelheim, Blade, Camurus, Corbus, DRG, EMD Serono, Eicos, Eiger, Guidepoint, Indalo, and Miysubishi. He has stock options in BriaCell and Pacific Therapeutics. 
SW was an employee of EMD Serono Inc. (a business of Merck KGaA, Darmstadt, Germany) at the time the study was conducted. He is now an employee of Neurogastrx, Inc.

CV-M, PF-M and DD are employees of EMD Serono Inc. (a business of Merck KGaA, Darmstadt, Germany).

CPD has received research grants from GlaxoSmithKline, CSF Behring, and Inventiva and consulting fees from EMD Serono, Roche/Genentech, Actelion, GlaxoSmithKline, Sanofi Aventis, Inventiva, CSL Behring, Boehringer-Ingelheim, Corbus and Bayer.

\section{Author appointments and qualifications}

Dinesh Khanna, Professor of Medicine at the University of Michigan, Scleroderma Program, Ann Arbor, MI, USA, MD, MS

Donald P Tashkin, Distinguished Emeritus Professor of Medicine at David Geffen School of Medicine at UCLA, Los Angeles, CA, USA, MD

Athol U Wells, Professor of Medicine and Consultant Chest Physician at Royal Brompton Hospital, London, UK, MD

James R Seibold, Principal Member of Scleroderma Research Consultants, LLC, Aiken SC, USA, MD

Stephen Wax, Senior Medical Director at EMD Serono Inc. (a business of Merck KGaA, Darmstadt, Germany), Billerica, MA, USA, at time of study. He is now Head of clinical research at Neurogastrx, Inc, $\mathrm{MD}, \mathrm{PhD}$

Cristina Vazquez-Mateo, Clinical Research Scientist at EMD Serono Inc. (a business of Merck KGaA, Darmstadt, Germany), Billerica, MA, USA, PhD

P Fleuranceau-Morel, Head of Safety Scientist at EMD Serono Inc. (a business of Merck KGaA, Darmstadt, Germany), Billerica, MA, USA, MBMS, PhD

Doris Damian, Director, Biostatistics at EMD Serono Inc. (a business of Merck KGaA, Darmstadt, Germany), Billerica, MA, USA, PhD 
Christopher P. Denton, Professor of Experimental Rheumatology at Royal Free Campus, University College London, U.K, PhD FRCP

\section{Address for correspondence}

Dr Dinesh Khanna

Division of Rheumatology

Department of Internal Medicine

University of Michigan Scleroderma Program

Ann Arbor, MI 48109

USA

Email:khannad@med.umich.edu

Phone: 001734.763 .7182

Running title: Abituzumab in SSc-ILD

Words: $1580 / 1500$

References: $12 / 20$

Figures + Tables: $3 / 3$ 


\section{ABSTRACT}

Objective. To investigate the effects of abituzumab in systemic sclerosis-associated interstitial lung disease (SSc-ILD).

Methods. STRATUS was a Phase II, double-blind, parallel-group, multicenter trial (NCT02745145). Adults ( $\leq 75$ years) with SSc-ILD on stable mycophenolate were randomized (2:2:1) to receive intravenous abituzumab $1500 \mathrm{mg}$, placebo, or abituzumab 500 mg every 4 weeks for 104 weeks. Primary endpoint: annual rate of change in absolute FVC. Results. STRATUS was terminated prematurely due to slow enrolment ( $\mathrm{n}=75$ screened, $\mathrm{n}=24$ randomized), precluding robust analysis of efficacy. Abituzumab was well-tolerated; no new safety signals were detected.

Conclusion. Further investigation of abituzumab for treatment of SSc-ILD is required.

\section{0 words}




\section{INTRODUCTION}

Systemic sclerosis (SSc) is a chronic, progressive autoimmune disease leading to abnormal fibrosis and vasculopathy of the skin and internal organs ${ }^{1}$. Pulmonary involvement particularly interstitial lung disease (ILD) - is the leading cause of death in $\mathrm{SSc}^{2,3,4}$. Preclinical models have provided robust evidence for the promotion of lung fibrosis by TGF $\beta^{5}$. TGF $\beta$ is activated by $\alpha v$-integrins on pulmonary epithelial cells ${ }^{6}$, making $\alpha v \beta 6$ a potential prognostic marker in $\operatorname{ILD}^{7,8}$. Blocking integrin $\alpha \mathrm{v}$ is therefore an attractive strategy for treating SSc-ILD. Abituzumab is a humanized monoclonal antibody previously evaluated in colorectal and prostate cancer ${ }^{10,11}$, that binds to and inhibits the activity of the $\alpha v$ integrin. The Phase II STRATUS study aimed to investigate the effects of abituzumab in patients with SSc-ILD receiving stable mycophenolate therapy but was terminated prematurely due to slow patient enrolment.

\section{METHODS}

STRATUS (NCT02745145; EMR200017-014) was a Phase II, randomized, double-blind, placebo controlled, parallel-group, multicenter trial of abituzumab in patients with SScassociated ILD conducted at 32 sites. The study was conducted in accordance with the ethical principles of the Declaration of Helsinki. The protocol was approved by the lead study site ethics board (Division of Rheumatology, University of Michigan, Ann Arbor, Michigan, United States; Approval Number: HUM00111923), as well as by the ethics boards of all other recruiting sites. The primary objective was to demonstrate efficacy of abituzumab in improving lung function in patients with SSc-ILD receiving background mycophenolate therapy. Secondary objectives included evaluation of safety and tolerability. 
Participants. Patients were men or women aged 18-75 who fulfilled the 2013 American College of Rheumatology/European League Against Rheumatism criteria for classification of SSc. Patients had to take a stable dose of 1.5-3 g/day mycophenolate mofetil (MMF) or 1080-2160 mg/day mycophenolate sodium (MPS) for $\geq 2$ months before screening and continue through the treatment period. The required duration of prior mycophenolate treatment was reduced from $\geq 6$ months to $\geq 2$ months following a protocol amendment.

Eligible patients had: $\geq 5 \%$ lung fibrosis on high-resolution computed tomography (HRCT; central reading) to enrich for risk of fibrotic progression; disease duration of $<7$ years from the first non-Raynaud's symptom; diffusing capacity of the lung for carbon monoxide (DLCO) $\geq 30 \%$ predicted; forced vital capacity (FVC) $40-85 \%$ predicted to ensure some reserve yet enrich for risk of progression and thus need for biologic therapy; and ratio of percent predicted FVC:DLCO $<1.8$ to ensure that individuals with a low likelihood of PH were selectively enrolled. All patients provided written informed consent and agreed to use robust contraceptive methods during the study.

Patients with underlying conditions constituting an inappropriate risk or contraindication for study participation (e.g. significant renal impairment, obstructive lung disease/emphysema, pulmonary hypertension, inflammatory connective tissue disease other than scleroderma-associated myopathy, fibromyalgia, and secondary Sjögren's syndrome, and/or tuberculosis) were excluded. Drugs other than MPS or MMF considered by the Investigator to have immunomodulating, immunosuppressive, or potential scleroderma disease-modifying properties were not permitted within 2 months before screening ( 5 months for cyclophosphamide). Medications for pulmonary arterial hypertension (e.g. endothelin receptor antagonists and phosphodiesterase type 5 inhibitors) were not permitted. 
Study design and endpoints. Patients were randomized (2:2:1) to receive intravenous (IV) infusions of abituzumab $1500 \mathrm{mg}$, placebo, or abituzumab $500 \mathrm{mg}$ every 4 weeks for 104 weeks (Figure 1). The primary endpoint was the annual rate of change in absolute FVC (mL), with the primary analysis conducted after all patients had completed 52-week study visit or discontinued the study treatment. Safety and tolerability were evaluated as: treatmentemergent adverse events (TEAEs), serious TEAEs, and treatment-emergent adverse events of special interest (AESIs); changes in clinical laboratory measures, electrocardiogram measures and vital signs; and incidence of patients with positive anti-drug antibody titres. Treatmentemergent AESI were defined as blistering, Grade 3+ 'skin and subcutaneous tissue disorders', or any cutaneous reaction of Grade 1-2 that worsened or did not respond to specific treatment.

\section{RESULTS}

Patient disposition. Planned enrolment was 175 patients, but the study was terminated early due to enrolment difficulties. Of 75 patients screened, 24 were randomized to treatment between November 2016 and January 2018 (abituzumab 1500 mg, n = 9; abituzumab 500 mg, $\mathrm{n}=5$; placebo, $\mathrm{n}=10)$. In total, $40(78 \%)$ screen failures reflected patients not meeting all eligibility criteria. The most common reason for screening failure was FVC $>85 \%$ predicted. Baseline characteristics are shown in Table 1.

All patients were withdrawn from study treatment before completion of the treatment period. For 19 patients $(79 \%)$ this was because of the sponsor's decision to terminate the study. Of the remaining 5 patients, 3 (13\%) had worsening of ILD, $1(4 \%)$ died (considered 
non-drug related) and 1 withdrew because of an adverse event (4\%). The median treatment duration was 25.9 weeks (abituzumab 1500 mg, 27.9 weeks; abituzumab 500 mg, 16.0 weeks; placebo, 26.6 weeks; range 8.0-64.1 weeks). Most patients (58\%) received 4-28 weeks of treatment, and $67 \%$ completed the administered infusions. Adherence to treatment (percentage of planned infusion volume received) was high (mean, 95\%), with 75\% of patients having $>80 \%$ adherence.

Efficacy. The number of patients enrolled precluded robust analysis of efficacy. The primary endpoint, however, was examined in line with the full study analysis plan. At baseline, mean (standard deviation) FVC was $2122 \mathrm{~mL}$ (449.0), $1958 \mathrm{~mL}$ (123.8) and $2117 \mathrm{~mL}$ (575.5) for the placebo, abituzumab $500 \mathrm{mg}$ and abituzumab $1500 \mathrm{mg}$ groups, respectively. Absolute changes from baseline in FVC over time are shown in Figure 2.

Safety. Overall, 21 of the 24 patients (88\%) experienced at least 1 TEAE. The most frequently occurring TEAEs were diarrhoea $(n=7 ; 29 \%)$ which lasted for a few days in most cases, and cough $(n=6,25 \%)$, which generally lasted for a few weeks or did not resolve, followed by fatigue, gastroenteritis, arthralgia, and headache, each occurring in 4 patients (17\%). There were no notable differences in the incidence of TEAEs between treatment arms. No TEAEs of special interest were reported (Supplementary Table 1). Three patients discontinued treatment because of TEAEs, 2 in the abituzumab $500 \mathrm{mg}$ arm (1 with fatal outcome [described below] and 1 with progressive ILD) and 1 in the placebo arm (non-serious cough and dyspnoea). Two additional patients discontinued treatment due to progressive disease, however, these cases were not reported as TEAEs leading to withdrawal. 
Four patients had serious TEAEs, none of which were considered related to study treatment. One patient receiving abituzumab $500 \mathrm{mg}$ had pneumonia, device-related infection (catheter for total parenteral nutrition) and multiorgan failure due to sepsis that led to death. One patient receiving placebo had pneumonia. One patient receiving abituzumab $1500 \mathrm{mg}$ had skin ulcer and 1 patient had small fibre neuropathy.

Observed changes from baseline in clinical laboratory values were not considered to be clinically relevant.

\section{DISCUSSION}

In the STRATUS study, abituzumab IV infusions of $500 \mathrm{mg}$ and $1500 \mathrm{mg}$ every 4 weeks were well-tolerated and treatment adherence was high. There were no new safety findings as compared with previous oncology trials ${ }^{10,11}$.

The study was terminated early due to low enrolment, and several factors contributed to this, including the rarity of SSc-ILD. While ILD affects $80 \%$ of patients with SSc, the global prevalence of SSc itself is only 1 in $10,000^{12}$. In addition, patients were initially required to have $\geq 6$ months of previous mycophenolate use, based on data from the SLS II study, in which MMF's effect on FVC was seen to be near maximal by $6-12$ months ${ }^{13}$. We hypothesized that requiring 6 months of previous mycophenolate would limit its confounding effect on the primary outcome measure. Mycophenolate uptake as standard-of-care was, however, slower than expected. Following slow recruitment, the protocol was amended to reduce mycophenolate therapy to $\geq 2$ months on a stable dose. Another factor was the concomitant recruitment into a large $(n=580)$ SSc-ILD study of nintedanib ${ }^{5,14}$, which may have been more attractive to patients and physicians, as nintedanib has proven efficacy in 
idiopathic pulmonary fibrosis ${ }^{15}$ and is taken orally (whereas abituzumab had not yet been evaluated in pulmonary fibrosis and STRATUS required intravenous infusions). Furthermore, the nintedanib study had broader inclusion criteria: patients could receive mycophenolate, methotrexate or no concomitant immunosuppressant, with no upper limit for $\mathrm{FVC}^{14}$.

The small patient numbers yielded few efficacy data points per timepoint. Only descriptive (not inferential) statistics were performed and, as expected with such a small sample size, no meaningful conclusions can be drawn.

In conclusion, abituzumab's effects on integrin $\alpha \mathrm{v}$ makes it a rational choice for SScILD treatment, particularly in cases with ongoing epithelial damage, since integrin $\alpha \mathrm{v}$ expression has been shown to be up-regulated in injured and inflamed epithelia, including in ILD. Targeting this integrin should be beneficial in individuals at risk of fibrotic progression. The STRATUS study enrolment therefore required both lung fibrosis on HRCT and decreased predicted FVC. The study was, however, terminated early following slow enrolment, likely due to multiple factors, including enrolment in other SSc trials, and eligibility criteria intended for enrichment of SSc-ILD patients at risk of progression and for reduction of variability of background medication (immunosuppressant). Abituzumab was well-tolerated with no new safety signals, but no efficacy conclusions can be drawn from the limited data obtained before termination.

\section{Acknowledgements}

The authors would like to thank the study patients and their families, the study investigators at each participating site, and study committee team members. Medical writing assistance was provided by Dan Booth and Hannah Fleetwood, Bioscript Group, Macclesfield, UK and 
supported by Merck KGaA, Darmstadt, Germany, in accordance with Good Publication Practice (GPP3) guidelines (http://www.ismpp.org/gpp3).

\section{REFERENCES}

1. Chowaniec M, Skoczynska M, Sokolik R, Wiland P. Interstitial lung disease in systemic sclerosis: Challenges in early diagnosis and management. Reumatologia 2018;56:249-54.

2. Giacomelli R, Liakouli V, Berardicurti O, Ruscitti P, Di Benedetto P, Carubbi F, et al. Interstitial lung disease in systemic sclerosis: Current and future treatment. Rheumatol Int 2017;37:853-63.

3. Silver KC, Silver RM. Management of systemic-sclerosis-associated interstitial lung disease. Rheum Dis Clin North Am 2015;41:439-57.

4. Strickland G, Pauling J, Cavill C, Shaddick G, McHugh N. Mortality in systemic sclerosis-a single centre study from the uk. Clin Rheumatol 2013;32:1533-9.

5. Khanna D, Tashkin DP, Denton CP, Lubell MW, Vazquez-Mateo C, Wax S. Ongoing clinical trials and treatment options for patients with systemic sclerosis-associated interstitial lung disease. Rheumatology (Oxford) 2019;58:567-79.

6. Katsumoto TR, Violette SM, Sheppard D. Blocking tgf $\beta$ via inhibition of the $\alpha v \beta 6$ integrin: A possible therapy for systemic sclerosis interstitial lung disease. Int J Rheumatol 2011;2011:208219.

7. Saini G, Porte J, Weinreb PH, Violette SM, Wallace WA, McKeever TM, et al. Avß6 integrin may be a potential prognostic biomarker in interstitial lung disease. Eur Respir J 2015;46:486-94.

8. Tatler AL, Jenkins G. Tgf-beta activation and lung fibrosis. Proc Am Thorac Soc 2012;9:130-6. 
9. Goodman SL, Picard M. Integrins as therapeutic targets. Trends Pharmacol Sci 2012;33:405-12.

10. Élez E, Kocáková I, Hohler T, Martens UM, Bokemeyer C, Van Cutsem E, et al. Abituzumab combined with cetuximab plus irinotecan versus cetuximab plus irinotecan alone for patients with kras wild-type metastatic colorectal cancer: The randomised phase i/ii poseidon trial. Ann Oncol 2015;26:132-40.

11. Hussain M, Le Moulec S, Gimmi C, Bruns R, Straub J, Miller K, et al. Differential effect on bone lesions of targeting integrins: Randomized phase ii trial of abituzumab in patients with metastatic castration-resistant prostate cancer. Clin Cancer Res 2016;22:3192200.

12. Denton CP, Khanna D. Systemic sclerosis. Lancet 2017;390:1685-99. 13. Tashkin DP, Roth MD, Clements PJ, Furst DE, Khanna D, Kleerup EC, et al. Mycophenolate mofetil versus oral cyclophosphamide in scleroderma-related interstitial lung disease (sls ii): A randomised controlled, double-blind, parallel group trial. Lancet Respir Med 2016;4:708-19.

14. Flaherty KR, Brown KK, Wells AU, Clerisme-Beaty E, Collard HR, Cottin V, et al. Design of the pf-ild trial: A double-blind, randomised, placebo-controlled phase iii trial of nintedanib in patients with progressive fibrosing interstitial lung disease. BMJ Open Respir Res 2017;4:e00212.

15. Richeldi L, du Bois RM, Raghu G, Azuma A, Brown KK, Costabel U, et al. Efficacy and safety of nintedanib in idiopathic pulmonary fibrosis. N Engl J Med 2014;370:2071-82. 
Tables and Figures

Table 1. Baseline demographic and disease characteristics.

\begin{tabular}{|c|c|c|c|}
\hline & $\begin{array}{l}\text { Placebo } \\
(n=10)\end{array}$ & $\begin{array}{l}\text { Abituzumab } 500 \mathrm{mg} \\
\qquad(\mathrm{n}=5)\end{array}$ & $\begin{array}{l}\text { Abituzumab } 1500 \mathrm{mg} \\
\qquad(\mathrm{n}=9)\end{array}$ \\
\hline Mean age, years (SD) & $51(11)$ & $60(8)$ & $55(8)$ \\
\hline \multicolumn{4}{|l|}{ Age category, $\mathrm{n}(\%)$} \\
\hline$<35$ years & $1(10)$ & $0(0)$ & $0(0)$ \\
\hline $35-49$ years & $3(30)$ & $0(0)$ & $2(22)$ \\
\hline 50-75 years & $6(60)$ & $5(100)$ & $7(78)$ \\
\hline Female, n (\%) & $8(80)$ & $4(80)$ & $7(78)$ \\
\hline \multicolumn{4}{|l|}{ Ethnicity, n (\%) } \\
\hline White (Hispanic or Latino) & $1(10)$ & $0(0)$ & $3(33)$ \\
\hline White (Other) & $9(90)$ & $5(100)$ & $6(67)$ \\
\hline \multicolumn{4}{|l|}{ Type of SSc, n (\%) } \\
\hline Diffuse cutaneous SSc & $6(60)$ & $1(20)$ & $7(78)$ \\
\hline Limited cutaneous SSc & $4(40)$ & $4(80)$ & $2(22)$ \\
\hline $\begin{array}{l}\text { Mean (SD) duration of SSc } \\
\text { (years) }\end{array}$ & $3.77(2.119)$ & $3.53(0.808)$ & $3.87(3.377)$ \\
\hline Mean (SD) QLF (\%) & $35.44(13.69)$ & $29.09(15.81)$ & $34.15(14.49)$ \\
\hline Mean (SD) FVC (mL) & $2122(449.0)$ & $1958(123.8)$ & $2117(575.5)$ \\
\hline $\begin{array}{l}\text { Mean (SD) DLCO } \\
(\mathrm{mmol} / \mathrm{min} / \mathrm{kPa})\end{array}$ & $3.3(0.72)$ & $3.2(0.95)$ & $4.1(1.23)$ \\
\hline
\end{tabular}

QLF: quantitative lung fibrosis (whole lung); SD: standard deviation; SSc: systemic sclerosis. 
Figure 1. STRATUS study design.

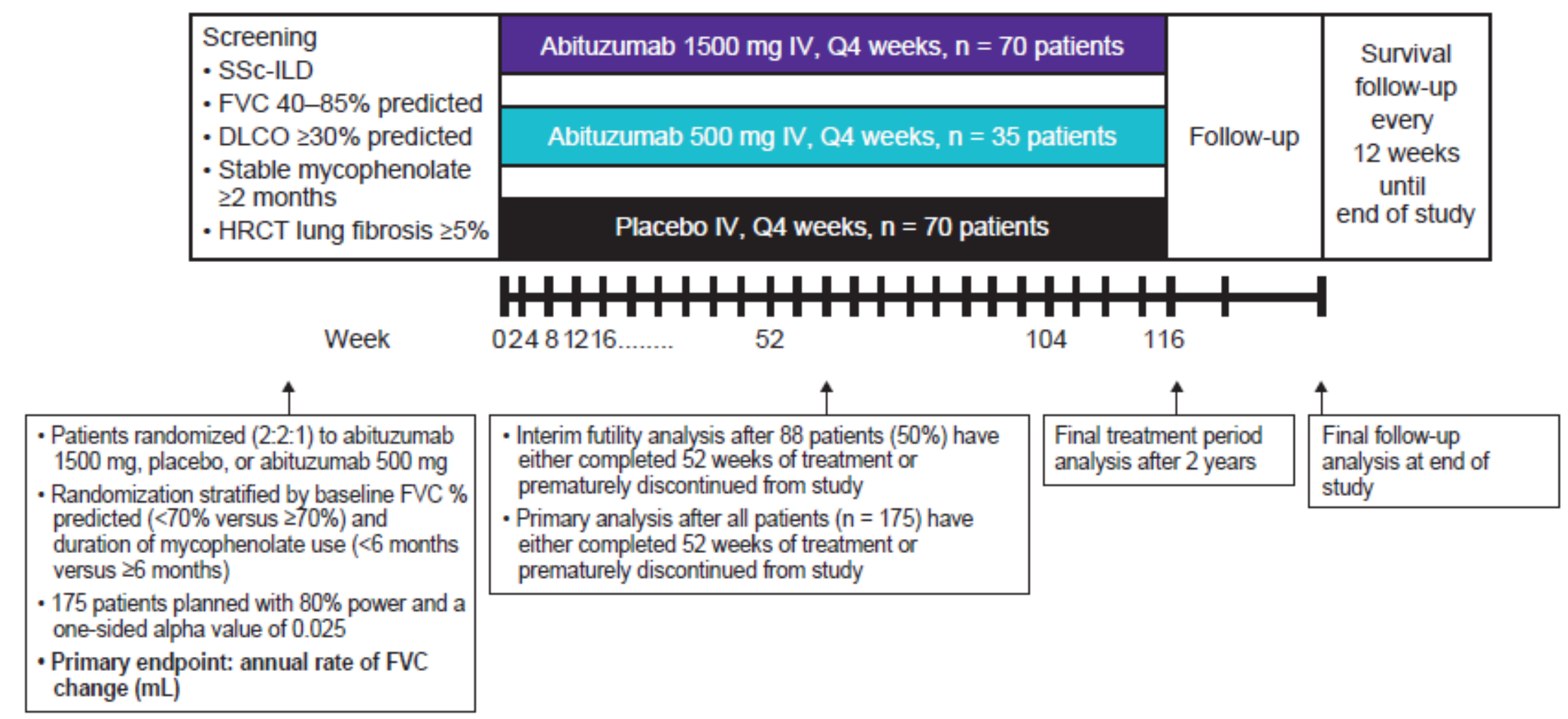

DLCO: diffusing capacity of lung for carbon monoxide; FVC: forced vital capacity; HRCT: high-resolution computed tomography; IV: intravenous; Q4 weeks: every 4 weeks; SSc-ILD: systemic sclerosis-associated interstitial lung disease. 
Figure 2. Mean absolute change from baseline in FVC over time, by treatment arm.

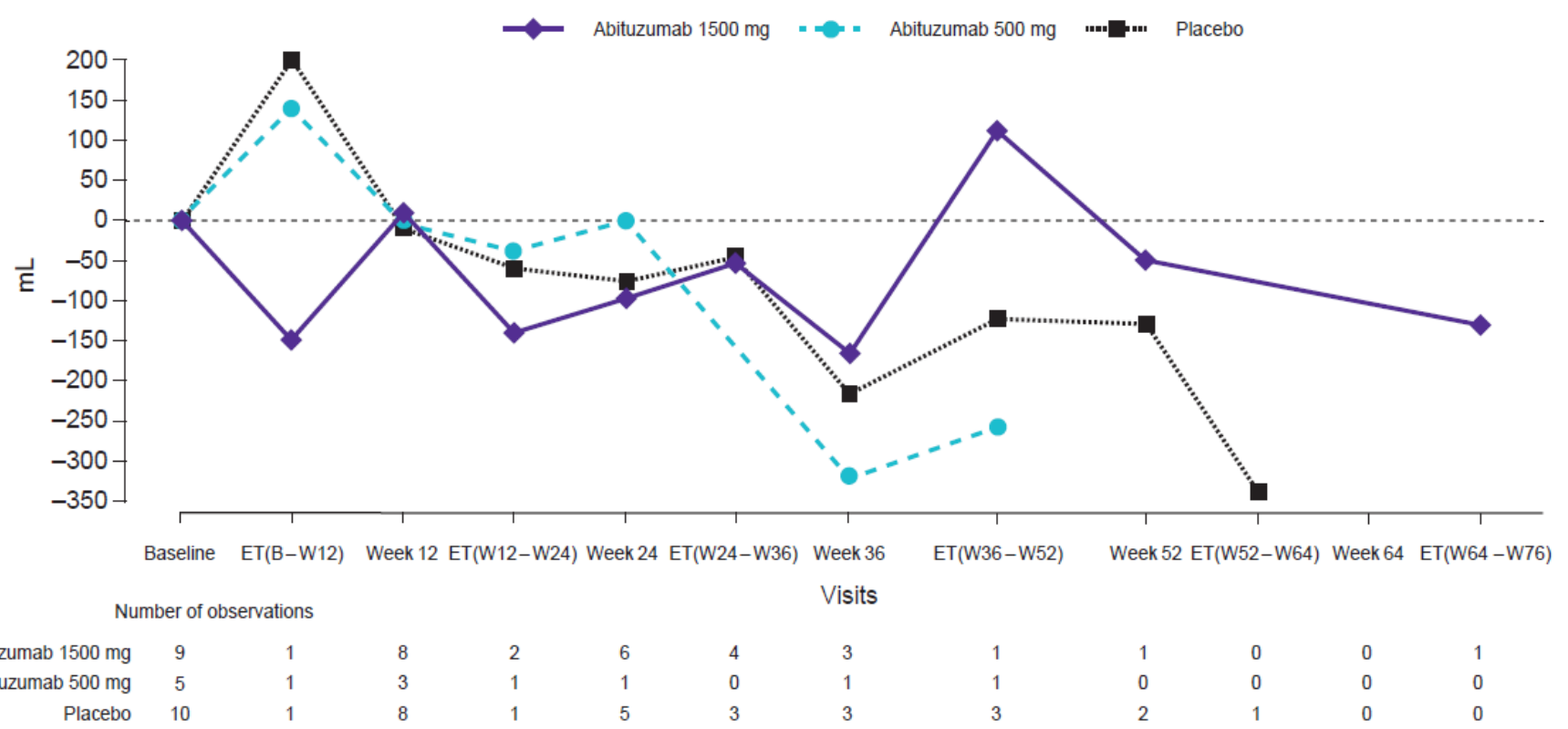

ET: early termination; FVC: forced vital capacity. 
Supplementary Table 1. Summary of TEAEs (safety analysis set).

\begin{tabular}{lcccc}
\hline & & Abituzumab & Abituzumab & \\
& Placebo & $500 \mathbf{~ m g}$ & $\mathbf{1 5 0 0} \mathbf{~ m g}$ & Total \\
$\mathbf{n}(\%)$ & $(\mathbf{n = 1 0})$ & $(\mathbf{n}=\mathbf{5})$ & $\mathbf{( n = 9 )}$ & $\mathbf{( n = 2 4 )}$ \\
\hline Any TEAE & $9(90)$ & $4(80)$ & $8(88.9)$ & $21(87.5)$ \\
Any serious TEAE & $1(10)$ & $1(20)$ & $2(22.2)$ & $4(16.7)$ \\
Any TEAE leading to death & $0(0)$ & $1(20)$ & $0(0)$ & $1(4.2)$ \\
Any treatment emergent AESI & $0(0)$ & $0(0)$ & $0(0)$ & $0(0)$ \\
Subjects with $\geq 1$ event & $9(90)$ & $4(80)$ & $8(88.9)$ & $21(87.5)$ \\
Most commonly occurring TEAEs & & & & \\
$\quad$ Diarrhoea & $3(30)$ & $1(20)$ & $3(33.3)$ & $7(29.2)$ \\
$\quad$ Cough & $3(30)$ & $0(0)$ & $3(33.3)$ & $6(25)$ \\
$\quad$ Fatigue & $1(10)$ & $1(20)$ & $2(22.2)$ & $6(16.7)$ \\
$\quad$ Gastroenteritis & $4(40)$ & $0(0)$ & $0(0)$ & $4(16.7)$ \\
Arthralgia & $1(10)$ & $1(20)$ & $2(22.2)$ & $4(16.7)$ \\
Headache & $1(10)$ & $1(20)$ & $2(22.2)$ & $4(16.7)$ \\
\hline
\end{tabular}

AESI: adverse event of special interest; TEAEs: treatment emergent adverse events. 